\title{
Magnetic resonance spectroscopy as a prognostic marker in neonatal hypoxic-ischemic encephalopathy: a study protocol for an individual patient data meta-analysis
}

\author{
Pieter LJ Degraeuwe ${ }^{1 *}$, Gerald J Jaspers ${ }^{1}$, Nicola J Robertson ${ }^{2}$ and Alfons GH Kessels ${ }^{3}$
}

\begin{abstract}
Background: The prognostic accuracy of ${ }^{1} \mathrm{H}$ (proton) magnetic resonance spectroscopy (MRS) in neonatal hypoxicischemic encephalopathy has been assessed by a criticized study-based meta-analysis. An individual patient data meta-analysis may overcome some of the drawbacks encountered in the aggregate data meta-analysis. Moreover, the prognostic marker can be assessed quantitatively and the effect of covariates can be estimated.

Methods: Diagnostic accuracy studies relevant to the study topic were retrieved. The primary authors will be invited to share the raw de-identified study data. These individual patient data will be analyzed using logistic regression analysis. A prediction tool calculating the individualized risk of very adverse outcome will be devised.

Discussion: The proposed individual patient data meta-analysis provides several advantages. Inclusion and exclusion criteria can be applied more uniformly. Furthermore, adjustment is possible for confounding factors and subgroup analyses can be conducted. Our goal is to develop a prediction model for outcome in newborns with hypoxic-ischemic encephalopathy.
\end{abstract}

Keywords: Hypoxic-ischemic encephalopathy, Infant-newborn, Magnetic resonance spectroscopy, Meta-analysis as topic

\section{Background}

Hypoxic-ischemic encephalopathy (HIE) in the newborn is associated with brain energy metabolism disturbances that can be quantified in vivo by ${ }^{1} \mathrm{H}$ (proton) magnetic resonance spectroscopy (MRS) [1,2]. A recent studylevel meta-analysis demonstrated that deep gray matter lactate/N-acetyl aspartate (Lac/NAA) peak/area ratio has a better prognostic accuracy than conventional and diffusion-weighted MRI for neurodevelopmental outcome after HIE [3].

The suggestion that Lac/NAA might support early clinical decisions was criticized for several reasons [4]. Although based on available studies, the validity of the meta-analysis and the generalizability of the results were

\footnotetext{
* Correspondence: pieter.degraeuwe@mumc.nl

'Department of Pediatrics, Maastricht University Medical Centre, P. Debyelaan 25, PO Box 5800, 6202AZ Maastricht, The Netherlands

Full list of author information is available at the end of the article
}

questioned [4]. It was suggested that the spectrum of the included patients was too broad, including very mildly (Sarnat 1 ) and very severely (Sarnat 3 ) affected patients; unclear selection criteria could undermine generalizability. In addition, some studies were restricted to surviving infants and the timing of the magnetic resonance (MR) studies varied. Death after HIE frequently follows decisions to withdraw life-sustaining care. Hence death as an adverse outcome may cause incorporation bias. It is conceivable that the outcome assessors were not always blinded to the MR results. Precise definitions of adverse outcome were lacking in studies. Concern was also expressed with respect to the post-hoc choice of 'cut-off values'. Wilkinson [4] concluded: 'It is not possible from published data to assess the usefulness of quantitative markers such as lactate/NAA peak/area ratio for predicting very adverse outcome $[\ldots]$ or to look

\section{Biomed Central}

(c) 2013 Degraeuwe et al.; licensee BioMed Central Ltd. This is an open access article distributed under the terms of the Creative Commons Attribution License (http://creativecommons.org/licenses/by/2.0), which permits unrestricted use, distribution, and reproduction in any medium, provided the original work is properly cited. 
separately at its usefulness for infants with moderate encephalopathy'.

An individual patient data (IPD) meta-analysis, where the raw data from multiple studies are synthesized, may overcome some of the drawbacks encountered in the aggregate data meta-analysis. A prerequisite is that all authors of the original studies are willing to share the individual test results and the patient characteristics to be evaluated. Subgroup analysis based on different HIE Sarnat stage is possible. Infants who died after treatment withdrawal can be excluded from the analysis. An agreed-upon definition of very adverse outcome can be applied uniformly. Finally, logistic regression modeling can be used to derive a prediction tool that calculates the individualized risk of very adverse outcome.

\section{Objectives}

The aims of the planned study are to reassess the prognostic performance of MR biomarkers in neonatal HIE and to determine the effect of other patient variables on the outcome using IPD meta-analysis. Logistic regression modeling will be used to develop a clinical risk prediction rule to assess the individual probability for adverse outcome after HIE.

\section{Methods}

Identification, selection, and appraisal of relevant studies have already been carried out independently, by two reviewers (PLJD, GJJ). Disagreement was resolved through discussion.

\section{Inclusion criteria for studies}

All studies, cohort and case-control studies, evaluating the prognostic accuracy of MRS biomarkers in term and near-term newborns with HIE (Sarnat stages 1, 2 and 3) were considered for review. Case-control studies are prone to spectrum bias [5], and the prevalence affects the predictive value or the post-test probability. Fortunately, in the regression equation:

$$
\operatorname{logit}(p)=\beta_{0}+\beta_{1} \cdot x_{1}+\beta_{2} \cdot x_{2}+\ldots+\beta_{n} \cdot x_{n}
$$

only $\beta_{0}$ is dependent on the prevalence. This constant $\beta_{0}$ can easily be readjusted to another (disease or) outcome prevalence.

The following data needed to be available for the study to be included in the IPD meta-analysis:

- MR spectroscopy data for Lac/NAA, Lac/creatine (Cr), Lac/choline (Cho), NAA/Cho, NAA/Cr, or Cho/Cr.

- Reliable, quantitative (numerical) neurodevelopmental outcome data at the age of at least 1 year (and preferably 2 years).

\section{Search strategy}

A systematic search was performed from inception until 14 April 2012 in MEDLINE (Ovid), EMBASE (Ovid), DARE [6], and Medion [7]. No diagnostic search filter $[8,9]$ or language restrictions were used. Details of the search are given in Additional file 1. Any duplicate articles identified were manually deleted. The reference lists of selected studies were checked for further relevant studies.

After removal of duplicates in our search (see Figure 1), we identified 203 studies of which 174 were excluded on the basis of title or abstract. Another three were excluded after assessment of the full text, and an additional three studies were identified through the reference lists, leaving 29 studies of which the authors will be contacted by email [10-38].

To confirm the thoroughness of our search, the authors will be asked whether they are aware of any untraced but eligible study.

\section{Quality assessment}

The quality of the 29 selected studies was assessed using the revised QUADAS tool [39,40]. Since MRS is an objective measurement and outcome is always assessed at a later stage, 3 of the 14 items in the QUADAS tool were omitted: time between index and reference test too long, blinded interpretation of the index test, and availability of clinical data. Two reviewers (PLJD, GJJ) independently answered the 11 remaining questions in the affirmative, in the negative or as being unclear. Figure 2 shows the quality of the 29 selected papers.

\section{Data items to be requested from the authors of the original diagnostic studies}

1. Gestational age at birth.

2. Birth weight.

3. Sex.

4. Apgar score at 1,5 , and 10 minutes.

5. Umbilical artery $\mathrm{pH}$ and base excess (or first arterial $\mathrm{pH}$ and base excess).

6. Sarnat score.

7. Was there an intrapartum sentinel event (for example, uterine rupture, placentalabruption, cord prolapse, and amniotic fluid embolism)?

8. Hypoglycemia: lowest blood (or plasma) glucose in the first 24 hours of life [41].

9. Hyperoxia and hypocarbia: highest arterial $\mathrm{pO}_{2}$ and lowest arterial $\mathrm{pCO}_{2}$ in the first day of life $[42,43]$.

10. Hypothermia (head or whole body cooling).

11. ${ }^{1} \mathrm{H}-\mathrm{MRS}$ biomarkers (timing, region of interest (ROI), echo time TE used): peak-area ratios of Lac/NAA, Lac/Cr, Lac/Cho, NAA/Cr, NAA/Cho, Cho/Cr. 


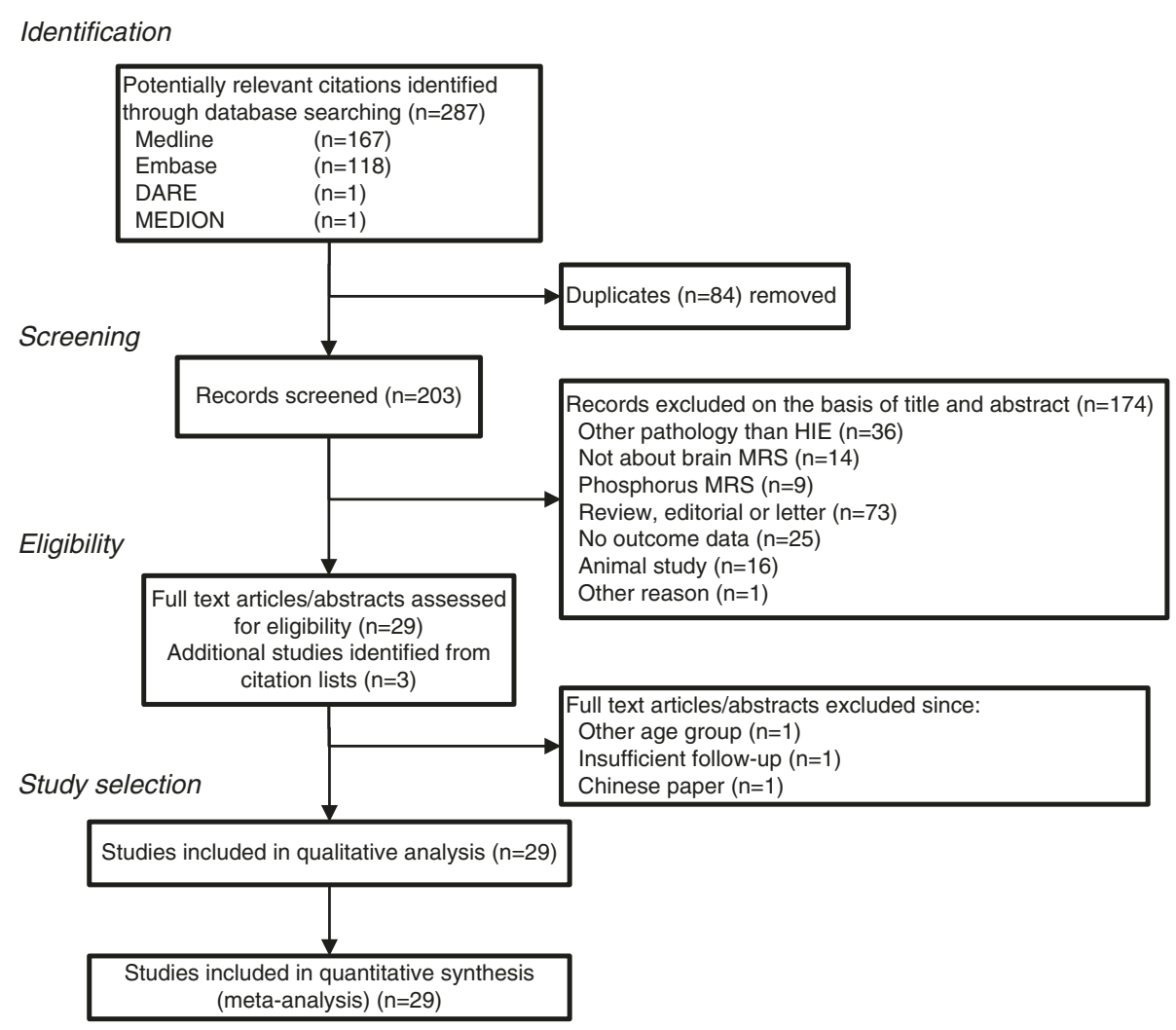

Figure 1 Flowchart showing search for and selection of papers evaluating prognostic value of ${ }^{1} \mathrm{H}-\mathrm{MRS}$ in neonatal hypoxic-ischemic encephalopathy. Final search carried out on 14 April 2012.

12. Outcome (at most recent assessment):

a. Motor function assessed by the Gross Motor Function Classification System (GMFCS) [44]. This five-level classification system describes the gross motor function of children and youths with cerebral palsy on the basis of their self-initiated movement with particular emphasis on sitting, walking, and wheeled mobility. A criteria list for scoring a child in different languages is available [45].

b. Early cognitive function as assessed by Griffiths Mental Developmental Scales (general cognitive quotient), scores on the Bayley II Mental Developmental Index or the Bayley III Cognitive

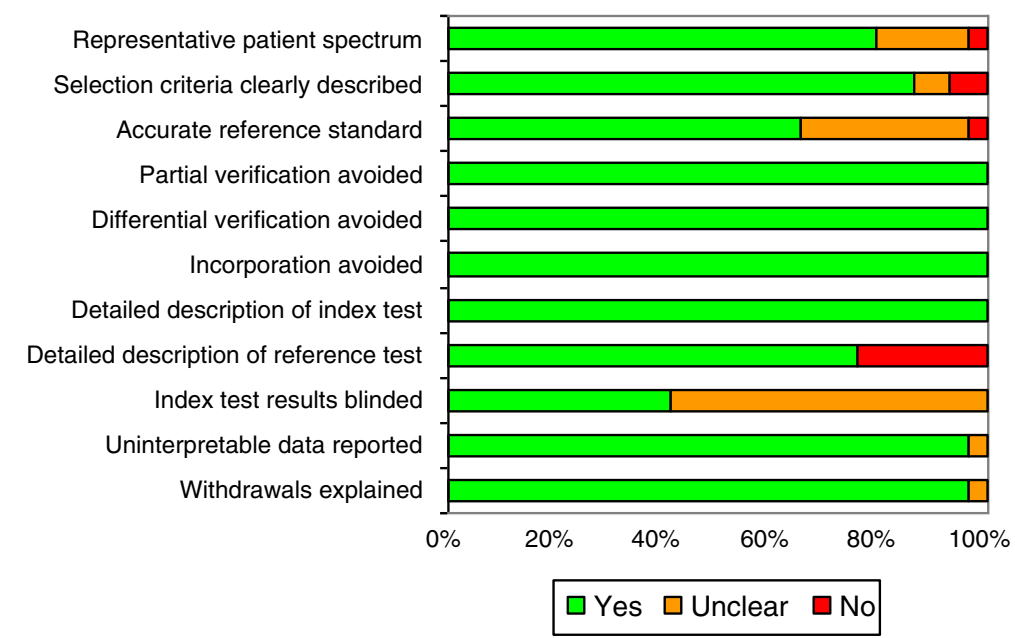

Figure 2 Results of quality assessment using QUADAS tool. 
Scale, or similar. For comparison, the raw scores will be converted to Z-scores to account for different standard deviations of the different test result distribution. Children who are too disabled for cognitive testing will be assigned a Z-score of -4 . Since early developmental testing is specific but not sensitive for later (school age) impairment [46], cognitive assessment at later age would improve the prognostic accuracy.

c. Visual: bilateral blindness.

d. Auditory: hearing loss requiring bilateral amplification.

13. Was death due to withdrawal of treatment?

The IPD will be delivered by the collaborating researchers using a spreadsheet form for completion in such a way that re-identification is impossible. The data set should not contain personal identifiers such as names, initials, addresses, ZIP code, phone numbers, date of birth or admission, medical record number, social security number, or other unique identifying numbers, characteristics, or codes.

\section{Definition of adverse outcome}

Adverse outcome will be defined as the presence of death, substantial motor dysfunction (severe motor impairment) with a level of III or worse on the GMFCS, bilateral blindness (or only light perception), and/or a developmental quotient of less than 3 standard deviations below the norm [47]. Alternative definitions can be used.

\section{Data synthesis and (statistical) analysis}

The MRS biomarkers of interest are Lac/NAA, Lac/Cr, Lac/Cho, NAA/Cho, NAA/Cr, and Cho/Cr. The contribution to the adverse prognosis of HIE of these biomarkers (as continuous variables) and the clinical characteristics will be explored using stepwise forward binary logistic regression analysis. The logit and logistic command in Stata/SE 10.1 will be used (Stata Corporation, College Station, TX, USA). An entry probability for each variable will be set at 0.05 . A clinical prediction rule will be derived from the final regression model. The predictive accuracy of the logistic regression models will be assessed by computing a cross classification table (lstat command) and by ROC curve analysis or c-statistics (roctab and roccomp command in Stata).

Between-study heterogeneity will be assessed by entering the study as a categorical variable in the logistic regression analysis.

The potential for publication bias will be estimated by using a Deeks' funnel plot. A $P$ value $<0.1$ was considered statistically significant [48].

\section{Planned sensitivity analyses}

The logistic regression analysis will be performed in surviving infants and in survivors plus infants whose decease was not due to a withdrawal decision.

\section{Ethical considerations}

The research will be conducted in accordance with the code of conduct for medical research of the Dutch Federation of Biomedical Scientific Societies [49].

The medical ethics committee at Maastricht University Medical Centre did not make an objection to the proposed IPD meta-analysis, but added the express condition that the patient data will be de-identified.

\section{Publication policy}

The results of the proposed IPD meta-analysis will be published on behalf of all researchers sharing a usable data set. At least one delegate per study will be proposed as co-author to the journals where the meta-analysis will be submitted. A larger study size may be represented by more authors. Alternatively, the paper will be published under a group name, allowing citation of all contributors in PubMed. The manuscript will be circulated to the collaborators for comments, amendments, and approval before being submitted.

The data provided by the authors will be treated confidentially and will not be copied or distributed elsewhere. Furthermore they will not be used for any other publication without the authors' approval.

\section{Registration}

The protocol has not been registered with PROSPERO (the international prospective register of systematic reviews).

\section{Discussion}

\section{Strength of the proposed study}

Individual participant data meta-analysis has been described as the 'gold standard' for prognostic accuracy studies [50]. In the proposed study, we hope to take advantage of several potential advantages of IPD metaanalysis [51,52]. Inclusion and exclusion criteria can be used more uniformly across studies, and overlapping sets of participants can be identified. Adjustments can be made for confounding factors, subgroup analyses can be conducted, and a prognostic model can be generated.

Important but challenging is the fact that follow-up information on neurodevelopmental outcome can be updated and uniformly described. To increase comparability between the studies, we hope the collaborators will be able to classify the motor outcome using the expanded and revised GMFCS [53]. Updated follow-up information on motor and cognitive developmental status will undoubtedly improve the validity of the prognosis. 


\section{Study weaknesses}

We are well aware that availability bias is inevitable. No meta-analysis can correct weaknesses in the contributing individual studies, such as diagnostic review bias [54].

Finally, heterogeneity due to different index test methodology (for example, ROI), and especially due to the use of different reference tests is inevitable [55,56], although for the latter problem conversion algorithms exist $[57,58]$. The modest predictive value of mental development assessment before the 2nd birthday for later cognitive status remains another concern [59].

\section{Summary}

Achieving a more uniformly defined outcome across studies, the possibility of obtaining longer-term outcome data, the exploration of interaction between MRS biomarkers and patient-level characteristics, and the possibility of sub-analyses, justify this proposed IPD metaanalysis.

\section{Additional file}

Additional file 1: Search strategies for the identification of studies: electronic searches.

\section{Abbreviations}

Cho: Choline; Cr: Creatine; GMFCS: Gross motor function classification system; HIE: Hypoxic-ischemic encephalopathy; IPD: Individual patient data; Lac: Lactate; MR: Magnetic resonance; MRI: Magnetic resonance imaging; MRS: Magnetic resonance spectroscopy; NAA: N-acetyl aspartate; ROI: Region of interest; TE: Echo time.

\section{Competing interests}

The authors declare that they have no competing interests.

\section{Authors' contributions}

PLJD conceived the study, identified, selected, and appraised the studies and drafted the manuscript. GJJ identified, selected, and appraised the studies. NJR contributed to the study design. AGHK gave methodological and statistical advice. All authors contributed to the critical revision of the manuscript. All authors have read and approved the final manuscript.

\section{Funding}

The writing of this study protocol had no explicit funding.

\section{Author details}

${ }^{1}$ Department of Pediatrics, Maastricht University Medical Centre, P. Debyelaan 25, PO Box 5800, 6202AZ Maastricht, The Netherlands. Department of Neonatology, UCL Institute for Women's Health, 86-96 Chenies Mews, WC1E $6 \mathrm{HX}$ London, England. ${ }^{3}$ Department of Clinical Epidemiology and Medical Technology Assessment, Maastricht University Medical Centre, PO Box 5800, 6202 AZ Maastricht, The Netherlands.

Received: 24 August 2013 Accepted: 11 October 2013 Published: 25 October 2013

\section{References}

1. Panigrahy A, Nelson MD Jr, Bluml S: Magnetic resonance spectroscopy in pediatric neuroradiology: clinical and research applications. Pediatr Radiol 2010, 40:3-30.

2. Robertson NJ, Cox IJ: Magnetic resonance spectroscopy of the neonatal brain. In MRI of the Neonatal Brain. 4th edition. Edited by Rutherford MA London: WB Saunders; 2002:295-313.
3. Thayyil S, Chandrasekaran M, Taylor A, Bainbridge A, Cady EB, Chong WK Murad S, Omar RZ, Robertson NJ: Cerebral magnetic resonance biomarkers in neonatal encephalopathy: a meta-analysis. Pediatrics 2010, 125:e382-e395.

4. Wilkinson D: MRI and withdrawal of life support from newborn infants with hypoxic-ischemic encephalopathy. Pediatrics 2010, 126:e451-e458.

5. Lijmer JG, Mol BW, Heisterkamp S, Bonsel GJ, Prins MH, van der Meulen JH, Bossuyt PM: Empirical evidence of design-related bias in studies of diagnostic tests. JAMA 1999, 282:1061-1066.

6. The University of York Centre for Reviews and Dissemination. [http://www.crd.york.ac.uk/crdweb/]

7. The Medion Database. [www.mediondatabase.nl]

8. Doust JA, Pietrzak E, Sanders S, Glasziou PP: Identifying studies for systematic reviews of diagnostic tests was difficult due to the poor sensitivity and precision of methodologic filters and the lack of information in the abstract. J Clin Epidemio/ 2005, 58:444-449.

9. Leeflang MM, Scholten RJ, Rutjes AW, Reitsma JB, Bossuyt PM: Use of methodological search filters to identify diagnostic accuracy studies can lead to the omission of relevant studies. J Clin Epidemiol 2006, 59:234-240.

10. Alderliesten T, De Vries LS, Benders MJNL, Koopman C, Groenendaal F: MR imaging and outcome of term neonates with perinatal asphyxia: value of diffusion-weighted MR imaging and ${ }^{1} \mathrm{H}$ MR spectroscopy. Radiology 2011, 261:235-242.

11. Amess PN, Penrice J, Wylezinska M, Lorek A, Townsend J, Wyatt JS, AmielTison C, Cady EB, Stewart A: Early brain proton magnetic resonance spectroscopy and neonatal neurology related to neurodevelopmental outcome at 1 year in term infants after presumed hypoxic-ischaemic brain injury. Dev Med Child Neurol 1999, 41:436-445.

12. Ancora G, Soffritti S, Lodi R, Tonon C, Grandi S, Locatelli C, Nardi L, Bisacchi N, Testa C, Tani G, Ambrosetto P, Faldella G: A combined a-EEG and MR spectroscopy study in term newborns with hypoxic-ischemic encephalopathy. Brain Dev 2010, 32:835-842.

13. Barkovich AJ, Baranski K, Vigneron D, Partridge JC, Hallam DK, Hajnal BL, Ferriero DM: Proton MR spectroscopy for the evaluation of brain injury in asphyxiated, term neonates. Am J Neuroradiol 1999, 20:1399-1405.

14. Bartha Al, Foster-Barber A, Miller SP, Vigneron DB, Glidden DV, Barkovich AJ, Ferriero DM: Neonatal encephalopathy: association of cytokines with MR spectroscopy and outcome. Pediatr Res 2004, 56:960-966.

15. Boichot C, Walker PM, Durand C, Grimaldi M, Chapuis S, Gouyon JB, Brunotte $F$ : Term neonate prognoses after perinatal asphyxia: contributions of MR imaging, MR spectroscopy, relaxation times, and apparent diffusion coefficients. Radiology 2006, 239:839-848.

16. Brissaud $O$, Chateil J-F, Bordessoules M, Brun M: Chemical shift imaging and localised magnetic resonance spectroscopy in full-term asphyxiated neonates. Pediatr Radiol 2005, 35:998-1005.

17. Cady EB: Metabolite concentrations and relaxation in perinatal cerebral hypoxic-ischemic injury. Neurochem Res 1996, 21:1043-1052.

18. Chateil JF, Quesson B, Brun M, Thiaudiere E, Sarlangue J, Delalande C, Billeaud C, Canioni P, Diard F: Localised proton magnetic resonance spectroscopy of the brain after perinatal hypoxia: a preliminary report. Pediatr Radiol 1999, 29:199-205.

19. Cheong JLY, Cady EB, Penrice J, Wyatt JS, Cox IJ, Robertson NJ: Proton MR spectroscopy in neonates with perinatal cerebral hypoxic-ischemic injury: metabolite peak-area ratios, relaxation times, and absolute concentrations. Am J Neuroradiol 2006, 27:1546-1554.

20. Groenendaal F, Van Der Grond J, Van Haastert IC, Eken P, Mali WPTM, De Vries LS: Results of cerebral proton magnetic resonance spectroscopy in neonates with asphyxia and neuromotor development. Ned Tijdschr Geneeskd 1996, 140:255-259.

21. Groenendaal F, Veenhoven RH, van der Grond J, Jansen GH, Witkamp TD, de Vries LS: Cerebral lactate and $\mathrm{N}$-acetyl-aspartate/choline ratios in asphyxiated full-term neonates demonstrated in vivo using proton magnetic resonance spectroscopy. Pediatr Res 1994, 35:148-151.

22. Hanrahan JD, Cox IJ, Azzopardi D, Cowan FM, Sargentoni J, Bell JD, Bryant DJ, Edwards AD: Relation between proton magnetic resonance spectroscopy within 18 hours of birth asphyxia and neurodevelopment at 1 year of age. Dev Med Child Neurol 1999, 41:76-82.

23. Hanrahan JD, Cox IJ, Edwards AD, Cowan FM, Sargentoni J, Bell JD, Bryant DJ, Rutherford MA, Azzopardi D: Persistent increases in cerebral lactate concentration after birth asphyxia. Pediatr Res 1998, 44:304-311. 
24. Kadri M, Shu S, Holshouser B, Deming D, Hopper A, Peverini R, Ashwal S: Proton magnetic resonance spectroscopy improves outcome prediction in perinatal CNS insults. J Perinatol 2003, 23:181-185

25. Khong PL, Tse C, Wong IYC, Lam BCC, Cheung PT, Goh WHS, Kwong NS, Ooi GC: Diffusion-weighted imaging and proton magnetic resonance spectroscopy in perinatal hypoxic-ischemic encephalopathy: association with neuromotor outcome at 18 months of age. J Child Neurol 2004, 19:872-881.

26. L'Abee C, De Vries LS, Van Der Grond J, Groenendaal F: Early diffusionweighted MRI and ${ }^{1} \mathrm{H}$-magnetic resonance spectroscopy in asphyxiated full-term neonates. Biol Neonate 2005, 88:306-312.

27. Leth H, Toft PB, Peitersen B, Lou HC, Henriksen O: Use of brain lactate levels to predict outcome after perinatal asphyxia. Acta Paediatr 1996, 85:859-864.

28. Meyer-Witte S, Brissaud O, Brun M, Lamireau D, Bordessoules M, Chateil JF: Prognostic value of MR in term neonates with neonatal hypoxicischemic encephalopath: MRI score and spectroscopy. About 26 cases. Arch Pediatr 2008, 15:9-23.

29. Miller SP, Newton N, Ferriero DM, Partridge JC, Glidden DV, Barnwell A Chuang NA, Vigneron DB, Barkovich AJ: Predictors of 30-month outcome after perinatal depression: role of proton MRS and socioeconomic factors. Pediatr Res 2002, 52:71-77.

30. Peden CJ, Rutherford MA, Sargentoni J, Cox IJ, Bryant DJ, Dubowitz LM: Proton spectroscopy of the neonatal brain following hypoxic-ischaemic injury. Dev Med Child Neurol 1993, 35:502-510.

31. Penrice J, Cady EB, Lorek A, Wylezinska M, Amess PN, Aldridge RF, Stewart A, Wyatt JS, Reynolds EO: Proton magnetic resonance spectroscopy of the brain in normal preterm and term infants, and early changes after perinatal hypoxia-ischemia. Pediatr Res 1996, 40:6-14.

32. Robertson NJ, Cowan FM, Cox IJ, Edwards AD: Brain alkaline intracellular $\mathrm{pH}$ after neonatal encephalopathy. Ann Neurol 2002, 52:732-742.

33. Robertson NJ, Cox IJ, Cowan FM, Counsell SJ, Azzopardi D, Edwards AD: Cerebral intracellular lactic alkalosis persisting months after neonatal encephalopathy measured by magnetic resonance spectroscopy. Pediatr Res 1999, 46:287-296.

34. Robertson NJ, Lewis RH, Cowan FM, Allsop JM, Counsell SJ, Edwards AD, Cox IJ: Early increases in brain myo-inositol measured by proton magnetic resonance spectroscopy in term infants with neonatal encephalopathy. Pediatr Res 2001, 50:692-700.

35. Roelants-Van Rijn AM, Van Der Grond J, De Vries LS, Groenendaal F: Value of ${ }^{1} \mathrm{H}-\mathrm{MRS}$ using different echo times in neonates with cerebral hypoxiaischemia. Pediatr Res 2001, 49:356-362.

36. Shanmugalingam $\mathrm{S}$, Thornton JS, Iwata O, Bainbridge A, O'Brien FE, Priest AN, Ordidge RJ, Cady EB, Wyatt JS, Robertson NJ: Comparative prognostic utilities of early quantitative magnetic resonance imaging spin-spin relaxometry and proton magnetic resonance spectroscopy in neonatal encephalopathy. Pediatrics 2006, 118:1467-1477.

37. Shu SK, Ashwal S, Holshouser BA, Nystrom G, Hinshaw DB Jr: Prognostic value of 1H-MRS in perinatal CNS insults. Pediatr Neurol 1997, 17:309-318.

38. Zarifi MK, Astrakas LG, Poussaint TY, Du Plessis A, Zurakowski D, Tzika AA: Prediction of adverse outcome with cerebral lactate level and apparent diffusion coefficient in infants with perinatal asphyxia. Radiology 2002, 225:859-870.

39. Whiting P, Rutjes AW, Reitsma JB, Bossuyt PM, Kleijnen J: The development of QUADAS: a tool for the quality assessment of studies of diagnostic accuracy included in systematic reviews. BMC Med Res Methodol 2003, $3: 25$.

40. Whiting PF, Weswood ME, Rutjes AW, Reitsma JB, Bossuyt PN, Kleijnen J: Evaluation of QUADAS, a tool for the quality assessment of diagnostic accuracy studies. BMC Med Res Methodol 2006, 6:9.

41. Tam EW, Haeusslein LA, Bonifacio SL, Glass HC, Rogers EE, Jeremy RJ, Barkovich AJ, Ferriero DM: Hypoglycemia is associated with increased risk for brain injury and adverse neurodevelopmental outcome in neonates at risk for encephalopathy. J Pediatr 2012, 161:88-93.

42. Klinger G, Beyene J, Shah P, Perlman M: Do hyperoxaemia and hypocapnia add to the risk of brain injury after intrapartum asphyxia? Arch Dis Child Fetal Neonatal Ed 2005, 90:F49-52.

43. Pappas A, Shankaran S, Laptook AR, Langer JC, Bara R, Ehrenkranz RA, Goldberg RN, Das A, Higgins RD, Tyson JE, Walsh MC: Hypocarbia and adverse outcome in neonatal hypoxic-ischemic encephalopathy. J Pediatr 2011, 158:752-758. e751
44. Palisano R, Rosenbaum P, Walter S, Russell D, Wood E, Galuppi B: Development and reliability of a system to classify gross motor function in children with cerebral palsy. Dev Med Child Neurol 1997, 39:214-223.

45. Barnett AL, Guzzetta A, Mercuri E, Henderson SE, Haataja L, Cowan F, Dubowitz L: Can the Griffiths scales predict neuromotor and perceptualmotor impairment in term infants with neonatal encephalopathy? Arch Dis Child 2004, 89:637-643.

46. GMFCS - Expanded and Revised (2007). [http://www.canchild.ca/en/ measures/gmfcs_expanded_revised.asp]

47. Lal M, Tin W: International perspectives: measuring perinatal outcomes why, when, and how: a British perspective. NeoReviews 2012, 13:e515-e526.

48. Deeks JJ, Macaskill P, Irwig L: The performance of tests of publication bias and other sample size effects in systematic reviews of diagnostic test accuracy was assessed. J Clin Epidemiol 2005, 58:882-893.

49. Abo-Zaid GMA: Individual patient data meta-analysis of prognostic factor studies, PhD thesis. University of Birmingham, School of Mathematics; 2011.

50. Code of Conduct for Medical Research. http://www.federa.org/sites/ default/files/bijlagen/coreon/code_of_conduct_for_medical_research_1.pdf.

51. Stewart LA, Tierney JF: To IPD or not to IPD? Advantages and disadvantages of systematic reviews using individual patient data. Eval Health Prof 2002, 25:76-97.

52. Riley RD, Lambert PC, Abo-Zaid G: Meta-analysis of individual participant data: rationale, conduct, and reporting. BMJ 2010, 340:c221.

53. Palisano RJ, Rosenbaum P, Bartlett D, Livingston MH: Content validity of the expanded and revised Gross Motor Function Classification System. Dev Med Child Neurol 2008, 50:744-750.

54. Begg CB: Biases in the assessment of diagnostic tests. Stat Med 1987, 6:411-423.

55. Anderson PJ, De Luca CR, Hutchinson E, Roberts G, Doyle LW: Underestimation of developmental delay by the new Bayley-III scale. Arch Pediatr Adolesc Med 2010, 164:352-356.

56. Vohr BR, Stephens BE, Higgins RD, Bann CM, Hintz SR, Das A, Newman JE, Peralta-Carcelen M, Yolton K, Dusick AM, Evans PW, Goldstein RF, Ehrenkranz RA, Pappas A, Adams-Chapman I, Wilson-Costello DE, Bauer CR, Bodnar A, Heyne RJ, Vaucher YE, Dillard RG, Acarregui MJ, McGowan EC, Myers GJ, Fuller J, Eunice Kennedy Shriver National Institute of Child Health and Human Development Neonatal Research Network: Are outcomes of extremely preterm infants improving? Impact of Bayley assessment on outcomes. J Pediatr 2012, 161:222-228. e223.

57. Lowe JR, Erickson SJ, Schrader R, Duncan AF: Comparison of the Bayley II mental developmental index and the Bayley III cognitive scale: are we measuring the same thing? Acta Paediatr 2012, 101:e55-58.

58. Ramsay M, Fitzhardinge PM: A comparative study of two developmental scales: the Bayley and the Griffiths. Early Hum Dev 1977, 1:151-157.

59. Sutcliffe AG, Soo A, Barnes J: Predictive value of developmental testing in the second year for cognitive development at five years of age. Pediatr Rep 2010, 2:e15.

doi:10.1186/2046-4053-2-96

Cite this article as: Degraeuwe et al:: Magnetic resonance spectroscopy as a prognostic marker in neonatal hypoxic-ischemic encephalopathy: a study protocol for an individual patient data meta-analysis. Systematic Reviews 2013 2:96.

\section{Submit your next manuscript to BioMed Central and take full advantage of:}

- Convenient online submission

- Thorough peer review

- No space constraints or color figure charges

- Immediate publication on acceptance

- Inclusion in PubMed, CAS, Scopus and Google Scholar

- Research which is freely available for redistribution 